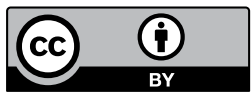

\title{
Participación comunitaria y percepción social en Latinoamérica: un futuro para las áreas protegidas y proyectos de geoparques*
}

\section{Community Participation and Social Perception in Latin America: a Future for Protected Areas and Geo-park Projects}

\section{J. L. Sánchez-Cortez**, M. C. Arredondo-García***, C. Leyva-Aguilera***, G. Ávila-Serrano*****, C. Figueroa-Beltrán ${ }^{* * * * * *}$ y J. M. Mata-Perellók****** Recibido: 2017-04-01 // Aprobado: 2017-05-12// Disponible en linea: 2017-06-30}

Cómo citar este artículo: Sánchez-Cortez, J. L., Arredondo-García, M. C., Leyva-Aguilera, C., Ávila-Serrano, G., Figueroa-Beltrán, C. y Mata-Perelló, J. M. (2017). Participación comunitaria y percepción social en Latinoamérica: un futuro para las áreas protegidas y proyectos de geoparques. Ambiente y Desarrollo, 21(41), 61-77. https://doi.org/10.11144/Javeriana.ayd21-41.pcps doi:10.11144/Javeriana.ayd21-41.pcps

\footnotetext{
* Los resultados de este artículo forman parte del proyecto Propuesta para generación y gestión de geoparques bajo estructuras de participación comunitaria en América Latina, realizado en el marco del programa de Doctorado en Medio Ambiente y Desarrollo de la Universidad Autónoma de Baja California, México. El proyecto, que se llevó a cabo entre abril del 2012 y enero del 2015, contó con el financiamiento de la Secretaría Nacional de Educación Superior, Ciencia y Tecnología (Senescyt-Ecuador) y del Consejo Nacional de Ciencia y Tecnología (Conacyt-México), además de la importante colaboración institucional del Ministerio del Ambiente (MAEEcuador), del Instituto Brasileño del Medio Ambiente y de los Recursos Naturales Renovables (Ibama-Brasil), de la Universidad de Carirí (Brasil), de la Comisión Nacional de Áreas Naturales Protegidas (Conanp-México) y de la Corporación Nacional Forestal (Conaf-Chile).

** Doctor en Medio Ambiente y Desarrollo, profesor de tiempo completo en la carrera de Ingeniería Ambiental, Universidad de Guayaquil, Ecuador. Correo electrónico: jose.sanchezco@ug.edu.ec. Autor de correspondencia.

*** Doctora en Oceanografía Costera, profesora titular de tiempo completo, Facultad de Ciencias Marinas, Universidad Autónoma de Baja California, México. Correo electrónico: conchita@uabc.edu.mx

**** Doctora en Medio Ambiente y Desarrollo, profesora titular de tiempo completo, Facultad de Ciencias, Universidad Autónoma de Baja California, México. Correo electrónico: cleyva@uabc.edu.mx Doctor en Geología, profesor titular de tiempo completo, Facultad de Ciencias Marinas, Universidad Autónoma de Baja California, México. Correo electrónico: gavila@uabc.edu.mx

****** Doctor en Medio Ambiente y Desarrollo, profesor de tiempo parcial, Facultad de Ciencias Marinas, Universidad Autónoma de Baja California, México. Correo electrónico: carlosfiguerioab@gmail.com

******* Doctor en Geología, magíster honoris causa de la Universidad Politécnica de Cataluña, España. Correo electrónico: rocpetrus@gmail.com
} 


\title{
Resumen
}

Las áreas naturales bajo régimen de protección han sido consideradas lugares con limitada participación comunitaria, especialmente en Latinoamérica, donde son evidentes los niveles de marginación y desigualdad. Los proyectos de geoparques fungen como alternativas vinculantes entre la sociedad y el patrimonio natural con base en las áreas protegidas ya existentes. En tal virtud, se levantó información social por medio de encuestas y observación directa en ocho territorios de cuatro países latinoamericanos: Brasil, Chile, Ecuador y México, con el propósito de examinar las condiciones de participación y percepción comunitaria. Los resultados muestran una similitud en los aspectos estimados para geoparques y áreas naturales protegidas (ANP) con respecto al fondo social. No obstante, se perciben temas como las motivaciones, la comunicación y la información que deben ser trabajados de manera intensiva para mejorar la participación y, a su vez, la percepción de las comunidades locales.

Palabras clave: participación comunitaria; geoparques; percepción social; América Latina; ANP

\begin{abstract}
The natural areas under a protection regime have been considered places with limited community participation, especially in Latin America, where the levels of marginalization and inequality are evident. The geo-park projects work as binding alternatives between society and the natural heritage based on the existing protected areas. In this way, we collected social information through surveys and direct observation in eight territories of four Latin American countries: Brazil, Chile, Ecuador, and Mexico. The purpose was to examine the conditions of participation and community perception. The results show a similarity in the estimated aspects for geo-parks and protected natural areas (PNA) with respect to the social fund. However, we capture topics such as motivations, communication, and information that must be worked intensively to improve participation and, in turn, the perception of local communities.
\end{abstract}

Keywords: community participation; geo-parks; social perception; Latin America; PNA 


\section{Introducción}

Desde su creación a finales del siglo XIX, las ANP se han considerado las estructuras de conservación más efectivas y extendidas en el planeta (Durand, 2010). Muchos de estos sitios se implementaron bajo un principio de exclusión, abandono de las relaciones o vínculos sociales, que se expresaban con restricciones y ausencia o limitación de vida humana y de sus actividades productivas (Rivas, 2006; Sánchez, 2011a), es decir, regidas por las dinámicas de los procesos naturales por fuera de las relaciones sociales. Esto, de alguna forma, deja de lado el verdadero sentido de la naturaleza y sus vínculos entre los diversos componentes, modifica los fenómenos y transforma las relaciones (Toledo, 2005).

Es necesario partir de la premisa de que los territorios guardan íntimas relaciones con la sociedad y su economía y, además, son el sustento de los elementos que se articulan para formar las identidades culturales (Leff, 2005). Así, también, son objeto de políticas que afectan directamente a las comunidades que dependen de dicho espacio (Vázquez Roca, 2010), incluso aquellas poblaciones humanas que tradicionalmente han habitado en las áreas naturales protegidas se consideraron amenazas para el equilibrio natural y fueron excluidas o expulsadas (Langton, Ma Rhea y Palmer, 2005).

Desde esta perspectiva, se complica el hecho de que las comunidades locales puedan participar o tomar decisiones sobre el manejo de los recursos naturales que les han sido limitados o apartado de sus alcances (Daim et al., 2012), tomando como partida que la participación social se encuentra estrechamente ligada al ejercicio de la democracia, acceso a la toma de decisiones y distribución de riqueza y poder, lo cual no se observa en un territorio bajo la denominación de ANP manejado por agentes externos (Rivas, López, Mosquera y Granizo, 2006).

Al conjugar los factores antes mencionados y la multiplicidad de matices existentes en relación con estas realidades, se obtiene como eminente resultado una percepción negativa de los territorios bajo protección genérica o específica (Man and the Biosphere Programme [MAB]-Organización de las Naciones Unidas para la Educación, la Ciencia y la Cultura [Unesco], 1978). Existe un divorcio entre la percepción inicial de un espacio y la apreciación de la misma área cuando es sometida a alguna nueva coyuntura para la conservación (Gerritesen, Monterio y Figueroa, 2003), ya que se ve afectada la perspectiva social con relación a la visión del manejo. En el mismo orden de cosas, en la actualidad un gran número de proyectos de geoparques en América Latina se sustenta en territorios sobre la base de ANP, aunque con una configuración conjunta y vinculante (Sánchez y Arredondo-García, 2012). En los geoparques la participación comunitaria pasa de ser una opción y se convierte en una necesidad (Unesco, 2010).

Para el desarrollo de este trabajo se realizó el levantamiento de información de campo, mediante técnicas de investigación social cualitativita y cuantitativa en seis áreas naturales protegidas, un geoparque de la Red Mundial y un proyecto de geoparque, ubicados en cuatro países latinoamericanos: Brasil, Chile, Ecuador y México, con el objeto de conocer los aspectos de participación e involucramiento de las comunidades aledańas e interiores a sus jurisdicciones.

En paralelo, la información alcanzada permitió estar al tanto de la percepción en los asentamientos rurales sujetos a encuestas y entrevistas, con relación al manejo del área, la gestión y la comunicación. Dado que los proyectos de geoparques en América Latina se encuentran en constante incremento (Mantesso-Neto et al., s. f.; Palacio, Sánchez y Schilling, 2016; Sánchez y ArredondoGarcía, 2012) y, a su vez, en los geoparques prevalece la condición comunitaria en el manejo (Sánchez, 2011a), se vuelve necesario considerar el profundizar en estrategias de participación que fortalezcan la percepción social y plantear proyectos de geoparques más sólidos y competitivos, verdaderos espacios no solo dedicados a la conservación, sino que también intervengan en las mejoras de la calidad de vida de las comunidades (Halim et al., 2011). 


\section{Metodología}

\section{Áreas de estudio}

Para el levantamiento de la información fueron considerados ocho territorios: geoparque Araripe en Brasil; proyecto de geoparque Kütralcura y reserva nacional Malalcahuello en Chile; parque nacional Machalilla, parque nacional Sangay y reserva faunística Chimborazo en Ecuador; y el parque nacional Constitución de 1857 y reserva de la biósfera El Pinacate en México (véase figura 1). Se trata de seis ANP, un geoparque de la red mundial y un proyecto de geoparque. Los sitios fueron elegidos de forma empírica debido a sus rasgos geológicos de importancia regional, sumado a que guardan relación con la iconología de conservación a escala nacional y, además, atienden a criterios geográficos por sus cercanías y facilidad de logística. En el caso del geoparque Araripe, se consideró el único geoparque latinoamericano incluido en la red mundial. Por otro lado, Kütralcura es un proyecto de geoparque con alta proyección.
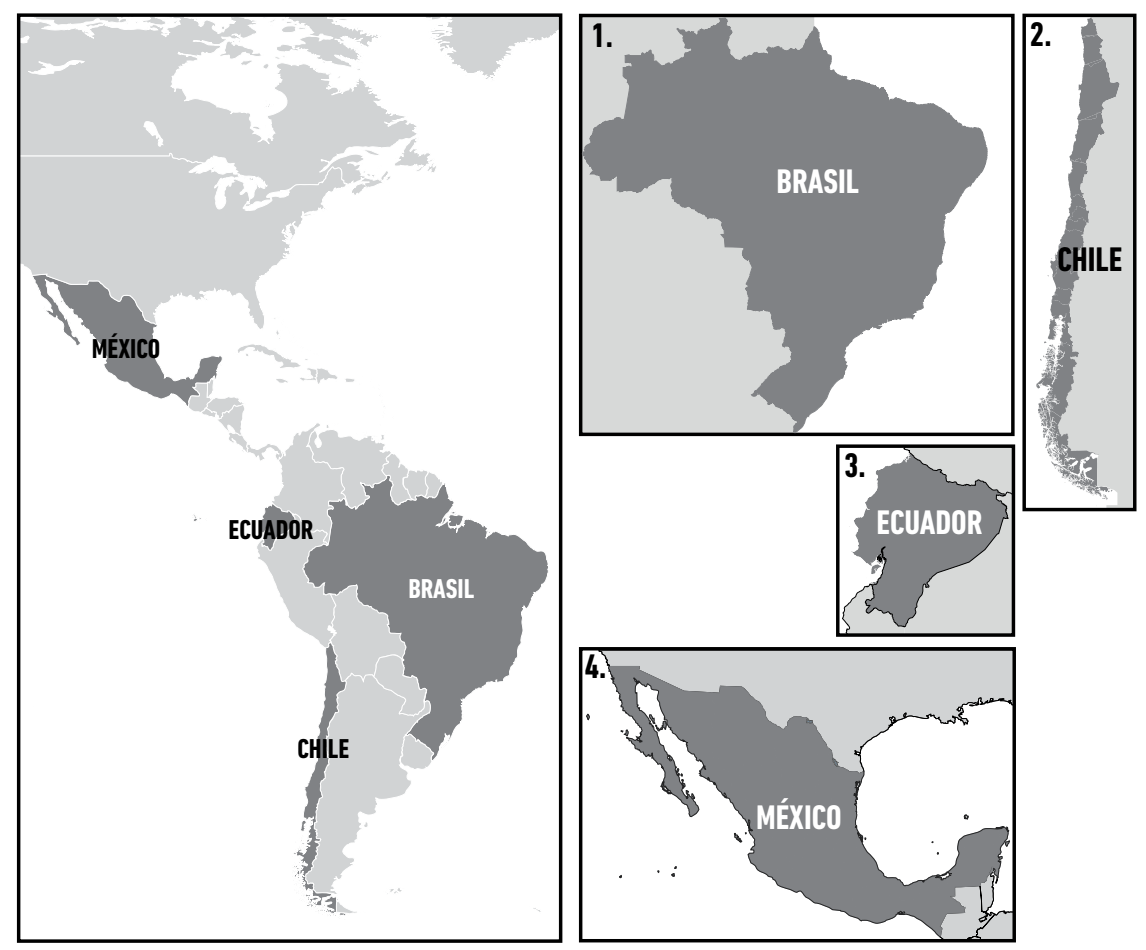

Figura 1. Ubicación de los países empleados como sitios de muestreo en América Latina: 1) Brasil, 2) Chile, 3) Ecuador y 4) México

Fuente: elaboración propia. 

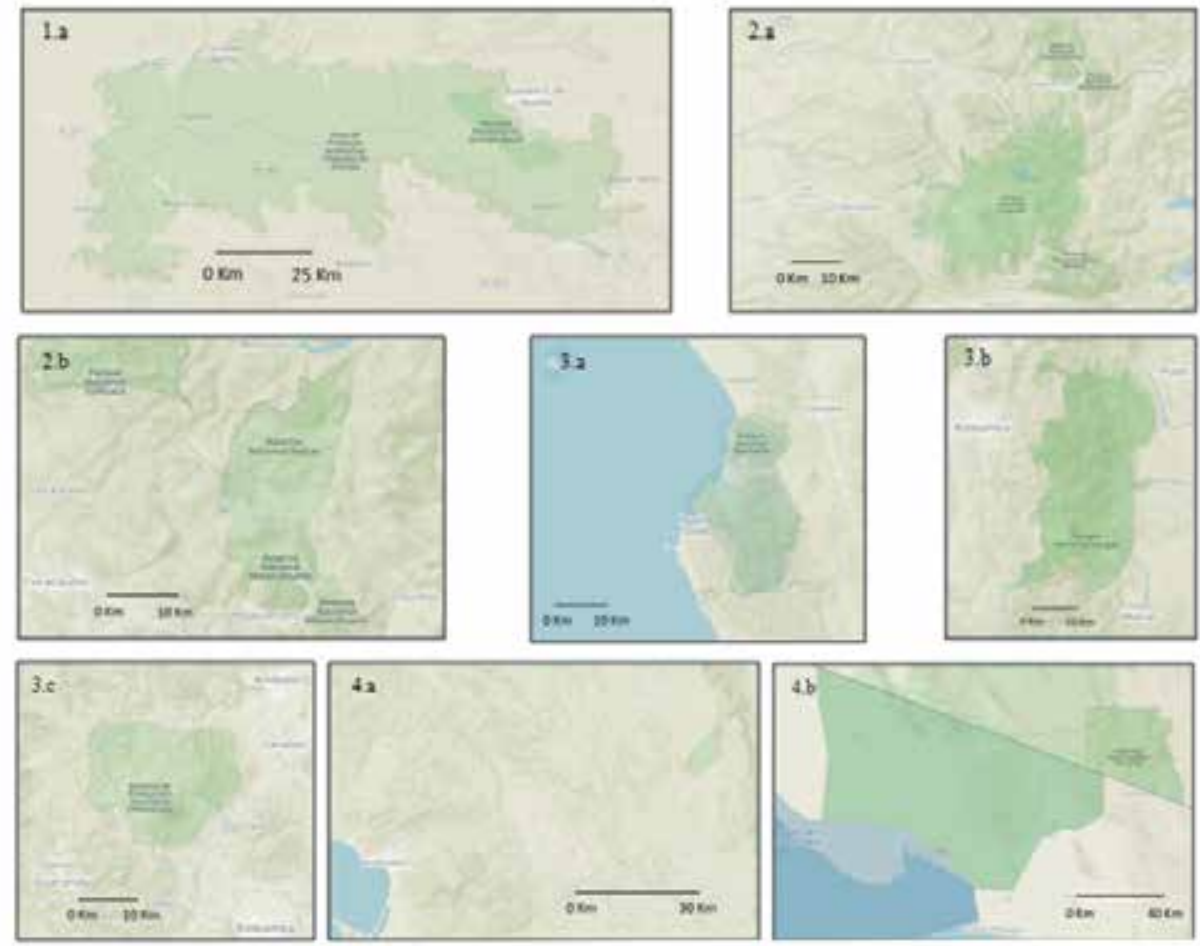

Figura 2. Ubicación de los sitios de muestreo en América Latina: 1) Brasil, 1a)

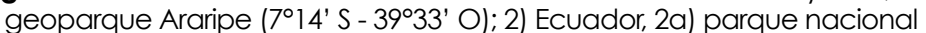

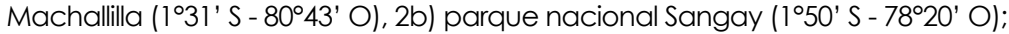
2c) reserva faunística Chimborazo (1⒉ $\left.28^{\prime} \mathrm{S}-7^{\circ} 49^{\prime} \mathrm{O}\right)$; 3) Chile, 3a) proyecto de

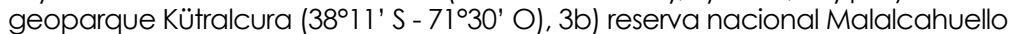

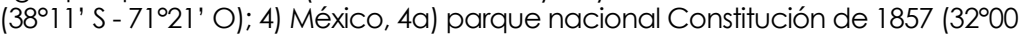
$\left.\left.\mathrm{N}-115^{\circ} 55^{\prime} \mathrm{O}\right), 4 \mathrm{~b}\right)$ reserva de la biósfera El Pinacate (31⒉ $\left.\mathrm{N}-112^{\circ} 59^{\prime} \mathrm{O}\right)$. Fuente: elaboración propia. 


\section{Aplicación metodológica}

Para el desarrollo de este trabajo se elaboró un cuestionario como instrumento de recolección de información, que consta de veintiún reactivos, de los cuales seis son respuestas abiertas y quince cerradas en escalas de Likert, para estar al tanto de la opinión, el conocimiento y la actitud con relación a las actividades en las que participan o han participado, así como su punto de vista de la conservación, la gestión, el manejo y la comunicación que ejerce el ente de conservación hacia la comunidad. La información de las encuestas fue complementada con los apuntes del diario de campo, asistido por observación directa de las personas encuestadas, además de entrevistas abiertas dirigidas a los administradores de cada ANP o geoparque, con el fin de integrar y triangular información para una mejor interpretación de los resultados obtenidos en las encuestas (Taylor y Bogdan, 1986).

El conjunto de preguntas de las encuestas se dividió en cuatro segmentos: perfil del encuestado, participación comunitaria, percepción comunitaria y actividades. La construcción del instrumento se realizó con base en los indicadores de evaluación para proyectos geoparques nacionales de la Red Mundial de Geoparques y manuales de evaluaciones rápidas para efectividad de manejo (Sistema Arrecifal Mesoamericano, 2004). El instrumento se aplicó, hasta cumplir con el tamańo de muestra, a personas mayores de quince ańos seleccionadas de forma aleatoria en las localidades interiores o aledańas a las ANP o geoparques, según el caso, buscando que la muestra fuera lo más representativa posible. Un primer cuestionario fue aplicado a veinticinco sujetos de las localidades de Ozogoche y Pumacoche, parque nacional Sangay, Ecuador, para los posteriores ajustes y aplicación final.

El tamaño de muestra en cada uno de los territorios fue definido mediante la fórmula de muestreo no probabilístico casual para poblaciones finitas (ecuación 1), al $95 \%$ de confianza y un error del $7 \%$, donde $\mathrm{N}$ es el número de habitantes (Morales Vallejo, 2008; Puma-Chávez, et al., 2011). Los valores de $\mathrm{N}$ para cada área se registran en la tabla 1 . Los resultados se presentan como porcentaje, con el fin de demostrar las frecuencias de respuestas en cada territorio sometido a investigación.

\begin{tabular}{ll}
\hline $\mathrm{n}=$ & Muestra \\
\hline $\mathrm{N}=$ & Población \\
\hline $\mathrm{e}=$ & Error $(7 \%)$ \\
\hline $\mathrm{z}=$ & Confianza $(95 \%)$ \\
\hline $\mathrm{p}=$ & $\begin{array}{l}\text { Proporción de respuestas. } 1 / 8 \\
=0.125\end{array}$ \\
\hline $\mathrm{q}=$ & $\begin{array}{l}1-\mathrm{p}=0.875 \\
\mathrm{n}=\frac{\mathrm{N}}{\mathrm{e}^{2}(\mathrm{~N}-1)} \\
\mathrm{Z}\end{array}$
\end{tabular}

(1) 
Tabla 1. ANP y geoparques, poblaciones, de acuerdo con la información de directores, administradores y coordinadores de cada área y su correspondiente de individuos para muestreo.

\begin{tabular}{llccc}
\hline \multicolumn{1}{c}{ País } & \multicolumn{1}{c}{ ANP o geoparque } & Área $\left(\mathrm{km}^{2}\right)$ & $N=$ Población & $n=$ Muestra \\
\hline Brasil & Geoparque Araripe & 3796 & 200000 & 86 \\
\hline Chile & Geoparque Kütralcura & 4200 & 18656 & 85 \\
\hline Chile & Reserva natural Malalcahuello & 127.89 & 900 & 78 \\
\hline Ecuador & Parque nacional Machalilla & 561.84 & 820 & 77 \\
\hline Ecuador & Parque nacional Sangay & 5177.65 & 1696 & 82 \\
\hline Ecuador & Reserva faunística Chimborazo & 585.60 & 1388 & 81 \\
\hline México & Parque nacional Constitución de 1857 & 50.09 & 2474 & 83 \\
\hline México & Reserva de la biósfera El Pinacate & 7146 & 58000 & 86 \\
\hline
\end{tabular}

Fuente: elaboración propia.

\section{Resultados y discusión}

Relación de los contextos de percepción y participación social en las ANP y Geoparque sometidos a análisis.

Se aplicaron 591 encuestas en los ocho territorios, dirigidas específicamente a pobladores permanentes. Conviene especificar que los datos y las características que se obtuvieron en este ensayo de campo corresponden únicamente a cada sitio que fue procesado, y no pueden generalizarse para cada nación. Casi un $70 \%$ de los encuestados son adultos, están en un rango entre 26 y 65 años; el $47.5 \%$ de mujeres y un $52.5 \%$ de hombres; es decir, existe una distribución más o menos equitativa en cuanto a los rangos de edades y género (tabla 2).

Más del $60 \%$ de los encuestados están conformes o satisfechos en algún grado con la administración. Así, se evidencia una percepción satisfactoria de la comunidad con relación a la conservación de la naturaleza en las áreas encuestadas, que se ha visto reflejada en los altos porcentajes de la muestra que consideran importante la preservación de los espacios protegidos cercanos a sus comunidades (tabla 2).

Los mayores porcentajes en cuanto a actividades económicas siempre fueron altos para las actividades que tienen relación con el usufructo de los recursos naturales, como la agricultura, la ganadería y la pesca. Tan solo en las ANP de México este valor estuvo muy por debajo de los demás sitios. Sin embargo, es información del noroeste de dicho país, y no necesariamente una condición generalizada a escala nacional. Por esta misma razón, se ha vuelto necesario el intercambio de acuerdos y reuniones entre administradores y la comunidad en general que permitan llegar a convenios sobre el uso de los recursos naturales.

Según el criterio de los administradores de cada una de las áreas protegidas en que se desarrolló este trabajo, los acuerdos afianzan el diálogo y la participación entre ambas partes, además, las comunidades pueden interceder directamente por su bienestar y se fortalecen las perspectivas entre comunidad y ANP. Para la interacción, ser flexibles es fundamental. Un administrador de un ANP en México manifestó: "han existido experiencias en las que se debe ceder las condiciones de amparo y protección de una ANP, con el fin de mantener estables las relaciones con los vecinos cercanos, evitar disputas y conflictos". 


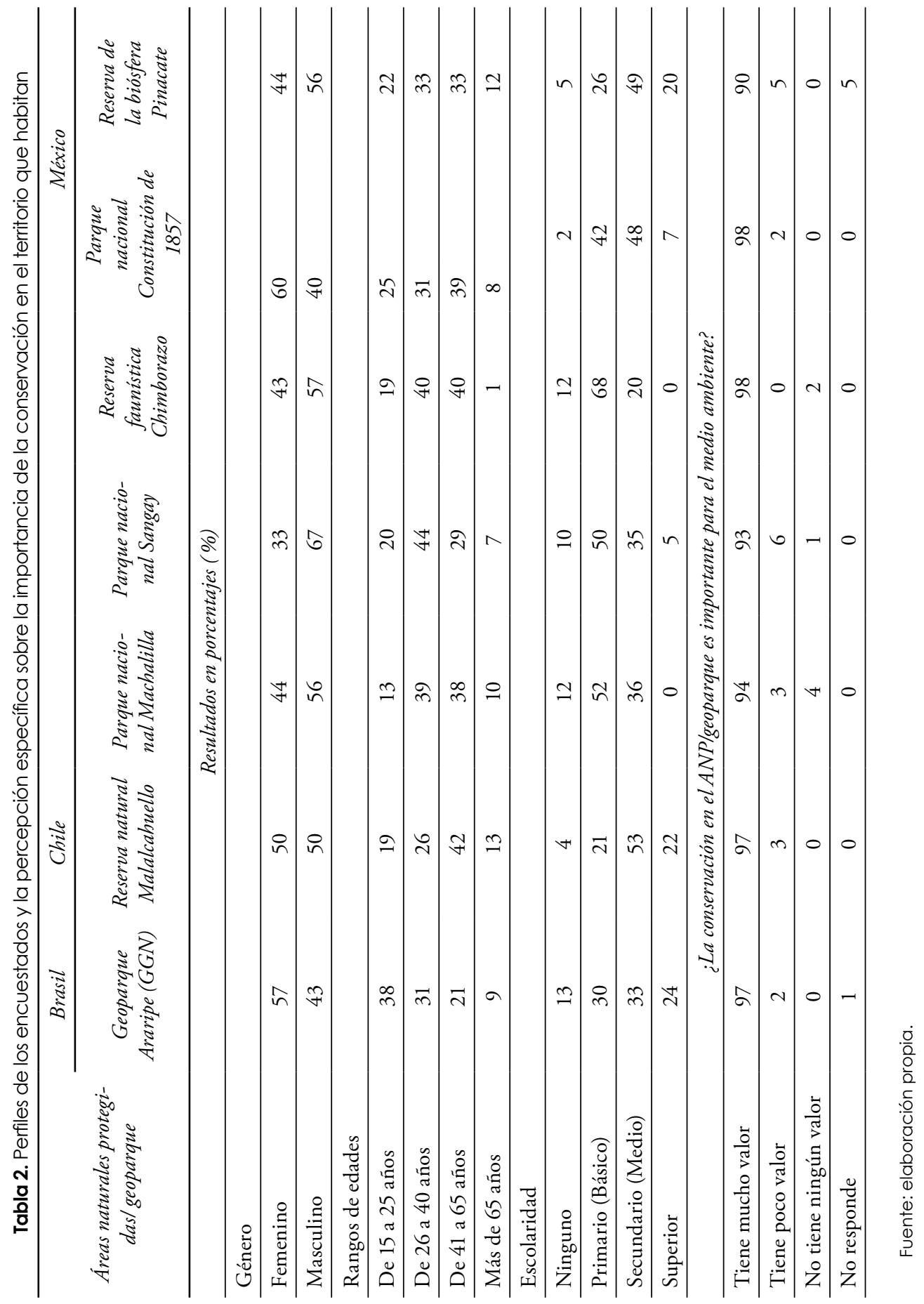




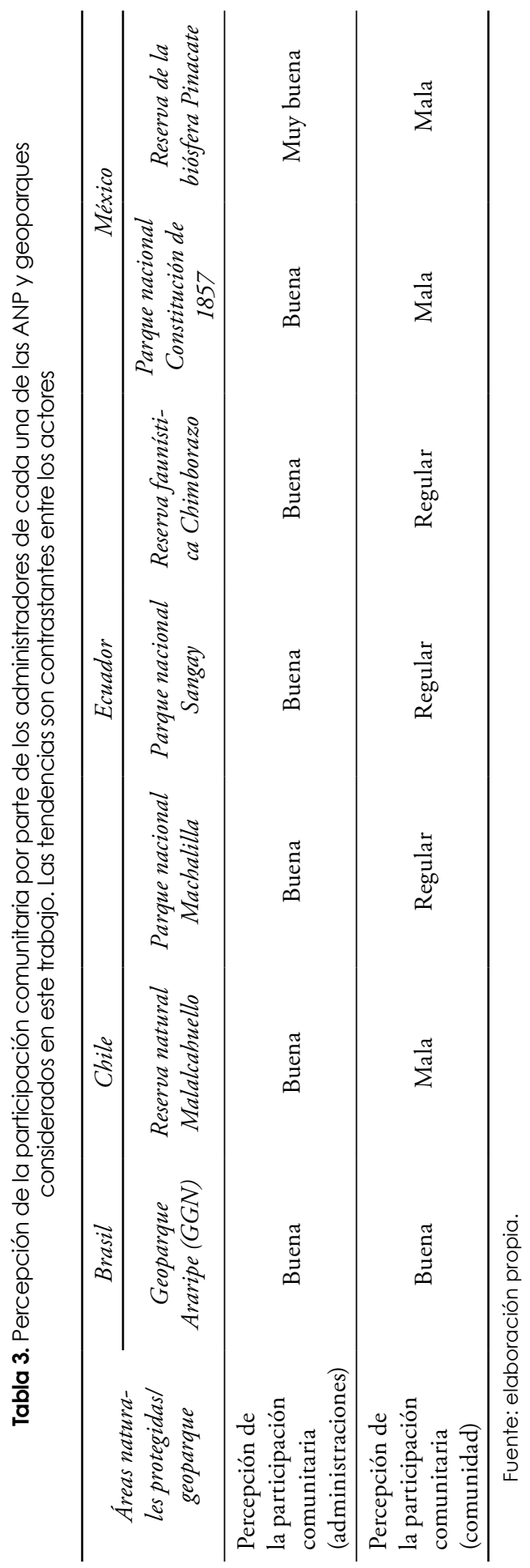




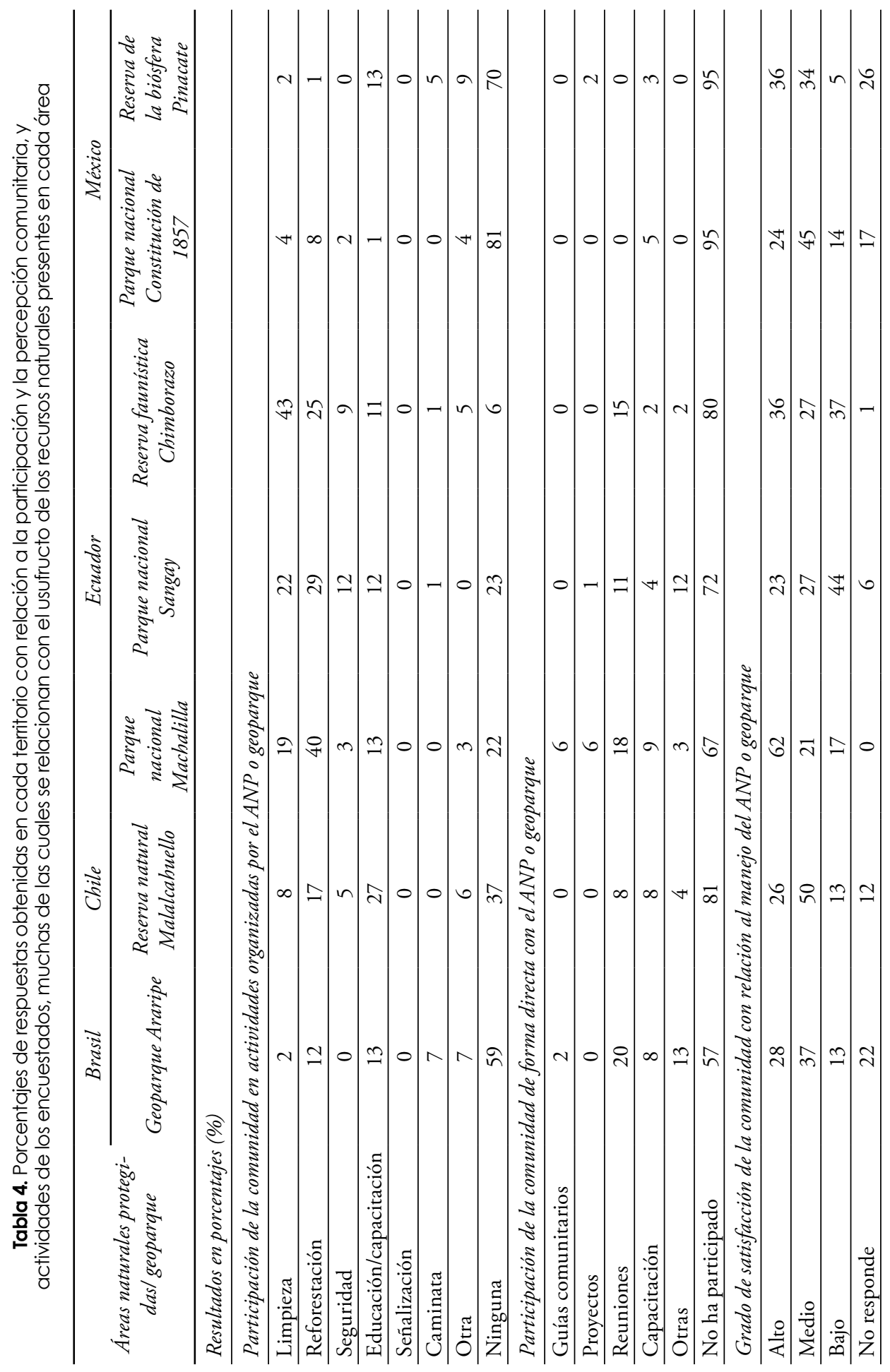




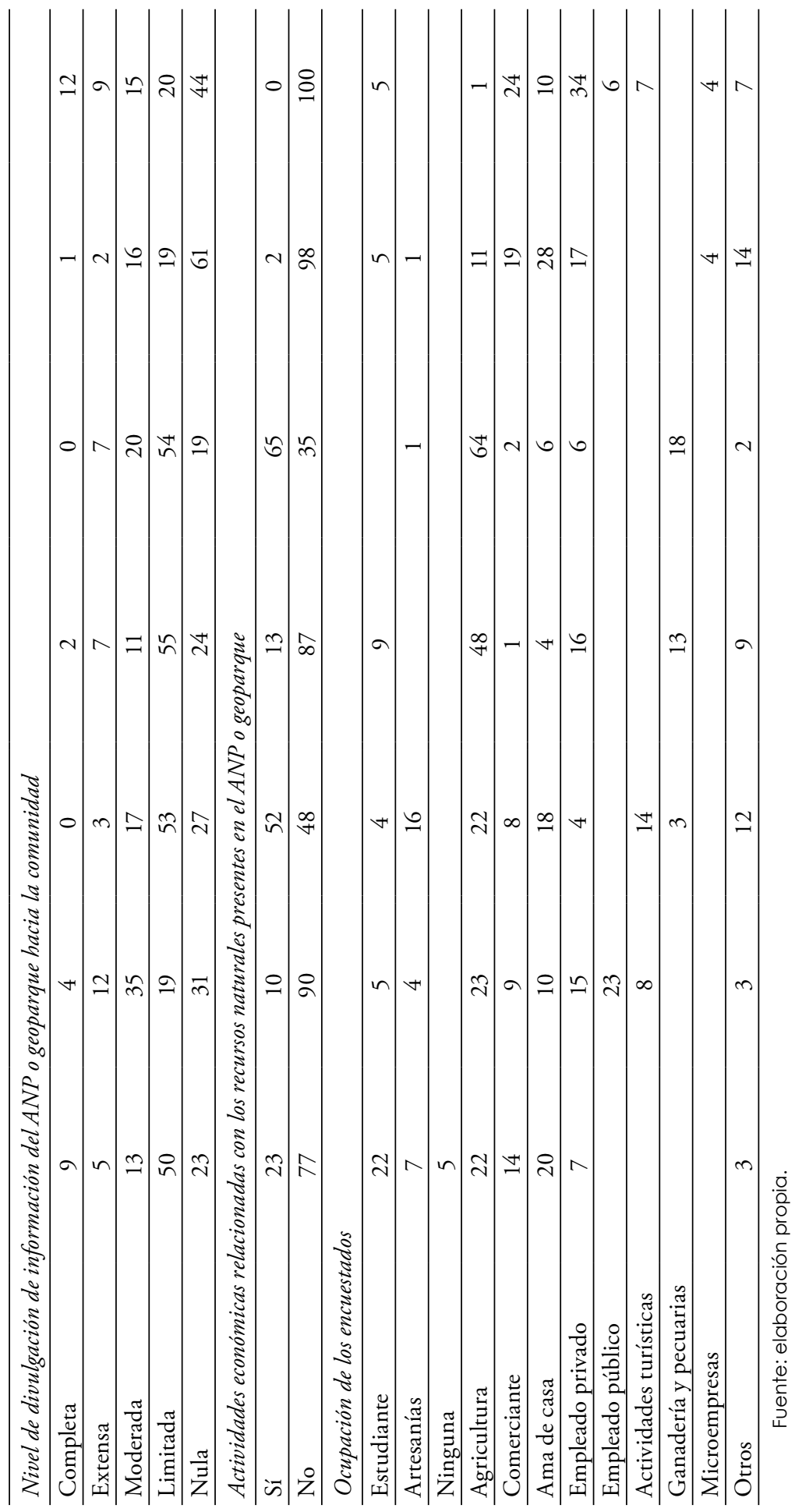


En la misma línea, la población encuestada expresó que participa en actividades organizadas por la administración, mas no se involucra directamente con ella; existe el deseo de participar, pero no el de hacer parte del manejo en un carácter formal, lo cual es contrastante, ya que en las entrevistas y en los cuestionarios dirigidos a las administraciones de cada área se destaca que las comunidades son partícipes en múltiples proyectos gestionados por los directivos y financiados principalmente por el Estado. Por otro lado, las perspectivas varían entre quien habita en el área bajo protección y la persona que protege la misma área (tablas 3 y 4 ).

Como muestra de lo antes dicho, el área de protección ambiental Chapada de Araripe, en CearáBrasil, cuenta con un consejo consultivo conformado por la comunidad, ONG y académicos, que suman un total de treinta integrantes, cuyas reuniones son cuatrimestrales. Desde mayo del 2008 a septiembre del 2011 se han llevado a cabo nueve convocatorias a consejo, de las cuales se han celebrado tres, debido a la falta de quórum de las seis reuniones restantes. El promedio de asistentes a dichas reuniones es de 33 personas, que representan al Gobierno Federal, a la Fiscalía Ambiental, el Ministerio del Ambiente y otras dependencias estatales, en una región en la cual la población bordea el millón de habitantes (Maier-Souza, 2012). De acuerdo con la autoridad, es probable que la falta de asistencia esté dada por las distancias que deben recorrerse para ir a dichas reuniones, o por problemas de comunicación de estas, aunque como bien se mencionó son de carácter ordinario cuatrimestral, sumado a que muchos de los sujetos del consejo tienen diferentes marcos interpretativos de acuerdo con sus diversos intereses en el ANP.

En el mediano plazo, las ANP efectivizan o agilizan la conservación una vez que son declaradas como tales, sin embargo, los geoparques son proyectos con resultados a largo plazo. Uno de los sitios en los que se levantó información fue el proyecto de geoparque Kütralcura en la Araucanía chilena, el cual viene desarrollándose desde mediados del año 2008, por conducto del Servicio Nacional de Geología y Minería de Chile (Sernageomin), y se considera uno de los proyectos de gran visión y perspectiva en la región (Mantesso-Neto, et al., s. f.; Palacio, Sánchez y Schilling, 2016; Schilling et al., 2010). No obstante, en casi diez años de trabajo, aún no se llega a toda la población involucrada en el proyecto, debido a que de 237 individuos a quienes se les consultó sobre alguna noción de este proyecto, tan solo 35 (14.8\%) mencionaron que habían escuchado noticias o comentarios sobre dicha intención, de los cuales 18 individuos $(7.6 \%)$ sabían de qué se trataba el proyecto de geoparque Kütralcura y especificaron que muchos de ellos eran empleados municipales o personas cuya actividad económica se relacionaba con el turismo.

La recolección de datos permitió obtener un marco regional que admite reconocer conductas de participación comunitaria en ANP, y a su vez estos puedan ser relacionados y comparables con geoparques y futuros proyectos de geoparques, aunque no se demuestra un patrón que indique diferencias relevantes entre cada componente o territorio analizado.

\section{Participación y percepción comunitaria en ANP y geoparques de América Latina como procesos sociales activos}

La participación es un proceso social dirigido a resolver necesidades fundamentales de los individuos, como un derecho aceptado y promovido por la sociedad que busca fortalecer mecanismos democráticos para formar parte de la toma de decisiones a diferentes escalas y ámbitos, mediante acuerdos y entendimientos. Tradicionalmente, los modelos de desarrollo en áreas protegidas se basan en una representación total del Estado (Liu, Ouyang, Miao, 2010). No obstante, ha sido ineludible superar barreras que permitan alcanzar el objetivo, pues en este proceso existe resistencia entre Estado y sociedad civil (Figueroa, 2003). A pesar de la intención, se continúa en el plano del discurso más que en la práctica (Doyon, 2008), ya que la mayor parte del tiempo los hábitos participativos se ven en la encrucijada del voluntarismo individual y la indiferencia con respecto a formar parte de estas experiencias colectivas. 
Una de esas barreras han sido las múltiples perspectivas de los individuos ante determinadas realidades, que son heterogéneas y a su vez variables y dependen de factores personales, sociales, culturales, políticos y económicos que, de alguna forma, determinan la percepción de un sujeto, así como los aspectos relacionados con el conocimiento, actos y decisiones. Las percepciones, visiones, relaciones, expectativas y demás son totalmente diferentes entre los tomadores de decisiones y la comunidad en general. Cuando existe el intercambio de experiencias, los resultados pueden vislumbrarse con percepciones y prioridades disímiles (Gerritesen, 2003).

Las áreas protegidas han sido consideradas por décadas las zonas con los mayores índices de pobreza y desigualdad (Riemann, et al., 2011). En virtud de ello, los gobiernos nacionales han desarrollado estrategias para aliviar la pobreza y la falta de recursos en comunidades rurales, donde la participación popular se ha convertido en un tema articulador, dada la necesidad de conquistar espacios por parte de la sociedad. Cada una de las naciones en las cuales se ha desenvuelto este trabajo, presenta estas alternativas para las comunidades rurales: Brasil Sin Miseria (Brasil), Chile Solidario (Chile), Socio Bosque/Socio Páramo (Ecuador), Programa de Empleo Temporal y Programa de Conservación para el Desarrollo Sostenible (México).

Aunque se trata de incentivos gubernamentales que promueven la participación entre Estado y sociedad, entre líneas se abre la posibilidad de democratizar la gestión de los recursos naturales. Las partidas presupuestales de estos programas sociales han sido benéficas inyecciones de capital económico en poblaciones tradicionalmente marginadas, sin embargo, el empoderamiento de estos proyectos por parte de la comunidad aún es difuso, ya que esta no los reconoce, como se muestra con el resultado de las encuestas. Si bien es cierto que las cifras en inversión social son reales, para la comunidad estas iniciativas se traducen en un trabajo jornal como cualquier otro (Cisneros y McBreen, 2010).

En contraste, el modelo de gestión participativa de un geoparque busca el empoderamiento social y puede fungir como un organismo que permita canalizar competentemente los instrumentos de apoyo para el desarrollo otorgados por los gobiernos de cada nación en la región (Socio Bosque, Socio Páramo, Programa de Empleo Temporal, etcétera), además de la posibilidad de autogestión mediante la creación de productos locales y el aprovechamiento sustentable de los recursos geológicos por medio del geoturismo. El caso específico de los geoparques se sustenta en tres ejes básicos: la conservación del medio ambiente, la educación en las ciencias de la tierra y, sobre todo, el fomento del desarrollo local (Mc Keever y Zouros, 2009), en el que la participación comunitaria, su conocimiento, artes, tradiciones y estilos de vida son vitales para el manejo y la gestión de un territorio bajo la denominación de geoparque (Mc Keever y Zouros, 2005; Sánchez, 2011b).

Desde su concepción, la gestión y la participación local son fundamentales en un proyecto de geoparque, para lo cual se requiere consolidar un fuerte apoyo de la comunidad, desarrollado a través de un proceso de abajo hacia arriba, en el que se involucre a autoridades, comunidades e inversionistas privados y, de la misma forma, instituciones educativas y de investigación (Unesco, 2010).

Un ejemplo que ilustra lo anterior es el geoparque de Langkawi, un pequeño archipiélago ubicado en Malasia, tradicionalmente dedicado a faenas de pesca y agricultura, en una zona fronteriza con Tailandia. A partir de 1987 el Gobierno declaró al archipiélago como una zona libre de impuestos, lo cual significó un crecimiento en relación con infraestructuras y facilidad de acceso a los servicios básicos. Esto asimismo generó una activación del espíritu empresarial local y el inicio de actividades turísticas, además de la recepción de subvenciones gubernamentales conocidas como Programa para el Desarrollo de la Región Económica del Corredor Norte.

En el año 2007 se obtuvo la aceptación por parte de la Red Mundial de Geoparques, para que Langkawi se considerara el primer geoparque malayo, hecho sin precedentes que potenció el turismo ecológico y el bienestar de las comunidades asentadas en el sitio, con base en el fortalecimiento de las industrias turísticas preexistentes y su diversificación hacia turismo ecológico, científico y educativo. 
Los habitantes isleños pasaron de ser pescadores artesanales a empresarios turísticos, sin perder su enraízo cultural (Azman et. al., 2010; Halim et. al., 2011).

A partir de datos y resultados, daría la impresión de que los geoparques efectivamente son sistemas de conservación incluyentes, participativos y vinculantes (Farsani, 2010), lo que se puede corroborar con su franco aumento y creciente interés. Tan solo desde octubre del 2009 hasta diciembre del 2012 se han adscrito 35 geoparques en la Red Mundial en tres años, lo cual ha significado que mejore una tendencia que se observó entre los años 2000 y 2009, periodo en el cual se incluyeron 58 geoparques en nueve años; en la actualidad suman 120 territorios.

A pesar de esta tendencia creciente, América Latina posee cuatro geoparques globales de Unesco, desde septiembre del 2006 a la actualidad. Dado que la región cuenta con las características geológicas, biodiversas y culturales circunscritas o afines a las áreas naturales protegidas, se espera una mayor representatividad en el futuro. Sin embargo, es probable que existan otros atributos que deban ser sujetos de evaluación para los futuros proyectos de geoparques (Sánchez, 2011b).

Los geoparques, a través de procesos participativos, buscan la implementación de un modelo de autogestión del territorio, por medio de una coordinación intersectorial e interinstitucional que permita mejorar la comunicación entre actores y alcanzar objetivos a mediano plazo. Esta evaluación muestra la necesidad de orientar prácticas de manejo más participativas, además de la optimización de las diferentes percepciones de la comunidad en una base territorial definida. Los proyectos de geoparques latinoamericanos enfrentan una nueva tendencia de conservación global. Además, se presentan como una alternativa viable para mejorar la calidad de vida de las comunidades rurales con un importante patrimonio natural, a través de la educación, la conservación y el fomento de la identidad, que a su vez fortalece el apego emocional y la valoración social del territorio.

\section{Conclusiones}

Con esta búsqueda de datos se aspira a crear un precedente que lleve a promulgar las ANP con un patrimonio geológico de nivel internacional. Los datos obtenidos cumplen el objetivo de comparar las particularidades de las áreas protegidas latinoamericanas con relación al geoparque Araripe, así como alcanzar una visión general de los niveles participativos en las diferentes áreas sujetas a investigación.

Las comunidades consideran de nula a limitada la comunicación que existe entre ellas y las ANP o geoparques, sin embargo, no hay una diferencia entre los resultados proyectados para ambos entes de conservación, lo que demuestra de alguna forma una calidad social, más que el sentido del instrumento de conservación o de manejo que se emplee para conservar. Fuera del componente netamente del patrimonio geológico, hablando de participación comunitaria, las características son básicamente similares entre geoparques y ANP latinoamericanas, y deben ser fortalecidas.

Las experiencias in situ, a partir de los diálogos sostenidos durante el proceso de investigación por observación directa, revelan la limitación de los métodos de comunicación empleados por ambos entes de conservación. En este punto en específico muchos encuestados manifiestan que, efectivamente, existe comunicación con los directivos o autoridades poblacionales, sin embargo, son los propios dirigentes comunales quienes no difunden la información receptada; hay recepción en la comunidad, aunque la transmisión aún es deficiente.

Para involucrar más actores al manejo de los recursos naturales, fuera del domino gubernamental, surge la necesidad de contar con interlocutores, grupos comunitarios, movimientos motivados (sin necesidad de estímulos económicos oficiales) para involucrarse en prácticas de participación de forma activa y distintiva, y romper así con el círculo de manejo privatizado del Estado. La participación social debe ser uno de los ejes básicos para replantear nuevas estrategias de conservación y protección de los recursos naturales, donde el aporte y la inclusión del concepto de geoparques en América Latina puede figurar como factor opcional para el logro de nuevos alcances, cada vez más participativos. 
Los resultados presentados en este trabajo corresponden a un grado de participación bajo y simbólico, de acuerdo con la escalera de participación ciudadana de Arnstein (1969). Es menester pensar en el perfeccionamiento y la optimización de las herramientas de participación comunitaria que pueden significar una contribución a un contexto renovado, en el que se articulen los diferentes valores sociales que predominen en una comunidad. El éxito de los procesos participativos debe basarse en la capacidad de las administraciones de brindar la oportunidad de consolidar una participación simbólica a una verdadera autogestión y poder ciudadano.

\section{Referencias}

Arntsein, S. (1969). A ladder of citizen participation. Journal of the American Planning Association, 35(4), $216-224$.

Azman, N., Halim, S. A., Liu, O. P., Saidin., S. y Komoo, I. (2010). Public education in heritage conservation for geopark community. Procedia Social and Behavioral Sciences, 7(C), 504-511.

Cisneros, P. y McBreen, J. (2010). Superposición de territorios indígenas y áreas protegidas en América del Sur. Quito: UICN.

Daim, M. S., Bakri, A. F., Kamarudin, H. y Zakaria, S. A. (2012). Being neighbor to a national park: are we ready for community participation? Procedia Social and Behavioral Sciences, (36), 211-220.

Doyon, S. (2008). La construcción social del espacio: el caso de la reserva de la biósfera de Ría Lagartos, Yucatán, México. En O. Beltrán, J. Pascual e I. Vacarro (Eds.), Patrimonialización de la naturaleza. El marco social de las politicas ambientales (pp. 289-308). Donostia: Ankulegi Antropologia.

Durand, L. y Jiménez, J. (2010). Sobre áreas naturales protegidas y la construcción de no-lugares. Notas para México. Revista Lider, 16, 59-72.

Farsani, N. T., Coelho, C. y Costa, C. (2010). Geoturism and geoparks as novel strategies for socio-economic development in rural areas. International Journal of Tourism Research, 13, 68-81.

Figueroa, J. (2003). Áreas naturales protegidas y la participación social en México. Academia Nacional de Educación Ambiental. Recuperado el 20 de mayo de 2013, de http://anea.org.mx.

Gerritesen, P., Monterio, M. y Figueroa, P. (2003). El mundo en un espejo. Percepciones campesinas de los cambios ambientales en el Occidente de México. Economía, Sociedad y Territorio, 4, 253-278.

Halim, S., Komoo, I., Salleh, H. y Omar, M. (2011). The geopark as a potential tool for alleviating community marginality. A case study of Langkawi Geopark, Malaysia. Shima: The International Journal of Research into Island Cultures, 1(5), 94-113.

Langton, M., Ma Rhea, Z. y Palmer, L. (2005). Community-oriented protected areas for indigenous peoples and local communities. Political Ecology, 12, 23-50.

Leff, E. (2005). La geopolítica de la biodiversidad y el desarrollo sustentable: economización del mundo, racionalidad ambiental y reapropiación social de la naturaleza. OSAL, 17, 263-273.

Liu, J., Ouyang, Z. y Miao, H. (2010). Community-oriented protected areas for indigenous peoples and local communities. Political Ecology, 12, 23-50.

Maier-Souza, P. F. (2012). APA Chapada do Araripe: Apresenta-se ao conselho. Crato-CE, Brasil: Instituto Chico Mendes de Conservação da Biodiversidade, Conselho Consultivo APA Chapada do Araripe.

Man and the Biosphere Programme (MAB)-Organización de las Naciones Unidas para la Educación, la Ciencia y la Cultura (Unesco). (1978). La perception de l'environnement: lignes directrices méthodologiques pour sur le terrain, Notes techniques du MAB 5, Preparé en coopération avec le Scope. París: autor. 
Mantesso-Neto, V., Mansur, K., López, R., Schilling, M. y Ramos, V. (s. f.). Geoparques en Latinoamérica. Recuperado el 1. ${ }^{\circ}$ de agosto de 2013, de http://www.sugeologia.org/documentos/ACTAS\%20VI\%20 CONGRESO\%20URUGUAYO/trabajos/105_Mantesso-Neto_Virginio.pdf

Mc Keever, P. y Zouros, N. (2005). Geoparks: celebrating earth heritage, sustaining local communities. Episodes, (28), 274-278.

Mc Keever, P. y Zouros, N. (2009). The Global Network of National Geoparks. Document presented in East Asia Geopark Conference, the East Asia Geopark Vision: 2009. Taipei, Taiwán.

Morales Vallejo, P. (2008). Tamaño necesario de la muestra: ¡cuántos sujetos necesitamos? En P. M. Vallejo (Ed.), Estadística aplicada a las ciencias sociales (pp. 2-24). Madrid: Universidad Pontífica Comillas.

Organización de las Naciones Unidas para la Educación, la Ciencia y la Cultura (Unesco). (2010). Directrices y criterios para Parques nacionales interesados en recibir asistencia de la Unesco para integrar la Red Mundial de Geoparques (GGN). París: Unesco, División de Ecología y Ciencias de la Tierra.

Palacio, J. L., Sánchez, J. L. y Schilling, M. (2016). Patrimonio geológico y su conservación en América Latina. Situación y perspectivas nacionales. México: Universidad Nacional Autónoma de México.

Puma-Chávez, A., Armijo-De Vega, C., Calderón-De La Barca, N., Leyva-Aguilera, J. y Ojeda-Benítez, S. (2011). Instrumento de evaluación para los programas de manejo de residuos domiciliarios. Investigación Ambiental, Ciencia y Politica Pública, 3(1), 18-29.

Riemann, H., Santes-Álvarez, R. y Pombo, A. (2011). El papel de las áreas naturales protegidas en el desarrollo local. El caso de la península de Baja California. Gestión y Política Pública, XX(1), 141-172.

Rivas, A., López, A., Mosquera, G. y Granizo, T. (2006). Participación social en el manejo de las áreas protegidas. Distribución de beneficios generados por la conservación de las áreas naturales protegidas. Una sistematización de las reflexiones en el Taller Regional organizado por The Nature Conservancy, 28 al 30 de junio de 2005. Quito: The Nature Conservancy.

Sánchez-Cortez, J. L. (2011a). Geoparques y áreas naturales protegidas: Una visión desde la conservación, identidad y participación social. Investigación Ambiental. Ciencia y Política Pública, 3, 44-51.

Sánchez-Cortez, J. L. (2011b). Geoconservación y geoparques en el contexto global: una visión holistica para América Latina y el Caribe. Documento presentado en el 2. Congreso Nacional de Geoturismo. San Felipe, Yaracuy, Venezuela.

Sánchez-Cortez, J. L. y Arredondo-García, M. (2012). Sudamérica: proyectos e iniciativas con miras hacia la creación de la Red Latinoamericana de Geoparques. Documento presentado en el VIII Reunión Nacional de Geomorfología, Guadalajara, México.

Schilling, M., Martínez, P., Toro, K., Contreras, P., Levy, C., Moreno, H., Naranjo, J. A. y Basualto, D. (2010). Territorial aspects in the establishment of the first national geopark in Chile. Document presented in European Geoparks Conference 2010. Langesund, Norway.

Sistema Arrecifal Mesoamericano. (2004). Manual para la evaluación rápida de la efectividad de manejo en áreas protegidas marinas de Mesoamérica. Ciudad de Guatemala: Proyecto para la conservación y uso sostenible del Sistema Arrecifal Mesoamericano. 
Taylor, S. J. y Bogdan, R. (1986). Introducción a los métodos cualitativos. La búsqueda de significados. Barcelona: Paidós.

Toledo, V. (2005). Repensar la conservación: ¿áreas naturales protegidas o estrategia bioregional? Gaceta Ecológica, 77, 67-83.

Vázquez Roca, A. (2010). El vértigo de la sobremodernidad: "no lugares", espacios públicos y figuras del anonimato. Nómadas, 16, 197-203. 